\title{
DESCRIPTION D'UNE NOUVELLE ESPECE DE TUBIFICIDAE DU NORD-OUEST DE L.'ESPAGNE : PROTUBERODRILUS TOURENQUI N. G., N. SP.
}

\author{
par N. GIANI ${ }^{1}$ et E. MARTINEZ̈-ANSEMIL"
}

Dans ce travail, nous décrivons une nouvelle espèce d'Oligochète récoltée dans la rivière Tambre au Nord-Ouest de l'Espagne. Protuberodrilus tourenqui n.g., n. sp. est un Tubificidae Rhyacodrilinae. Il se distingue nettement de tous les autres représentants de cette sous-famille par la forme longuement pédonculée des atria ainsi que par la présence d'un porophore mâle, unique, très développé. De plus, il possède des soies copulatrices portées au niveau de chaetophores saillants sur les segments IX, X et XII. Il est d'ailleurs le seul Tubificidae à présenter des soies génitales modifiées sur un segment post-atrial.

\section{Description of a new species of tubificid from the North West of Spain : Protuberodrilus tourenqui n. g., n. sp.}

We describe a new species of oligochaete collected from the river Tambre in the North West of Spain. Protuberodrilus tourenqui n. g., n. sp. belongs to the Tubificidae: Rhyacodrilinae. It is clearly separated from all other members of this sub-family by the long pedunculated form of the atria as well as by the presence of a unique and well-developed male porophore. In addition, it has copulatory chaetae at the level of the chaetophores projecting from segments IX, $X$ and XII. It is furthermore the only tubificid to have modified genital chaetae on a post-atrial segment.

Au cours d'une étude écologique sur les Oligochètes du Tambre, réalisée par l'un d'entre nous (Martinez-Ansemil, en préparation), nous avons récolté plusieurs exemplaires d'un nouveau représentant de la famille des Tubificidae (Oligochaeta). Nous en donnons ici la description.

\section{1. - STATION DE RECOLTE}

Nous avons rencontré cet Oligochète dans la rivière Tambre qui coule en direction est-ouest dans la région de Galice au nord-ouest de l'Espagne avant de se jeter dans l'océan Atlantique à Noya. Les récoltes ont été effectuées à proximité de Sobrado de los Monjes, à une altitude de $480 \mathrm{~m}$. A cette station le Tambre a une largeur de $5 \mathrm{~m}$,

1. Laboratoire d'Hydrobiologie, Université Paul Sabatier, 118, route de Narbonne, 31077 Toulouse Cedex.

2. Colegio Universitario de Orense, C. General Franco, 35 - Orense (Espagne). 
une profondeur de 0,2 à $0,8 \mathrm{~m}$. Le substrat est formé de galets placés sur un fond de graviers et de sable grossier, recouverts par endroits par des touffes d'Oenanthe. Les prélèvements ont été effectués dans des zones à courant rapide. Cette station sera décrite en détail dans notre travail en préparation (loc. cit.).

Ce nouvel Oligochète était présent dans 3 prélèvements effectués au filet de Surber, le 27 janvier 1979. Ce jour-là la température était de $6,8{ }^{\circ} \mathrm{C}$, le $\mathrm{pH}$ de 7 et la conductivité de $33 \mu \mathrm{ohm} / \mathrm{cm} / \mathrm{cm}^{2}$; l'eau était saturée en oxygène.

\section{2. - DESCRIPTION DE L'ESPECE}

\subsection{Matériel}

La description a été effectuée uniquement sur des spécimens fixés au formol : 4 individus matures et un immature qui possédait déjà certaines soies génitales modifiées. L'étude a été faite sur le matériel suivant :

- 2 individus montés in toto dans du polyvinyl-lactophénol ;

- 1 individu coupé au microtome à $7 \mu$ d'épaisseur ;

- 2 individus disséqués et montés au baume du Canada après coloration par l'hématoxyline acide d'Erlich.

\subsection{Morphologie externe}

FORME ET TAILLE

- Longueur du corps (matériel fixé) : 1,2 à $2,4 \mathrm{~cm}$;

- Diamètre au niveau du segment $\mathrm{V}: 0,5 \mathrm{~mm}$; au niveau du segment XI : 0,55 à $0,67 \mathrm{~mm}$ (cette mesure comprend la hauteur du porophore mâle) ;

- Nombre de métamères variant de 60 à 70 environ ;

- Epiderme (25 à $30 \mu \mathrm{m}$ ) dépourvu de papilles ;

- Prostomium conique et pointu, aussi long que large : 165 à $225 \mu \mathrm{m}$.

LES SOIES

- Formule sétale :

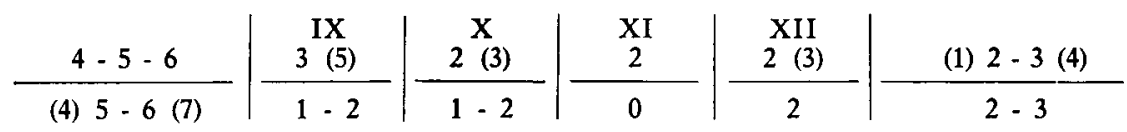

\section{- Soies normales}

Il n'y a pas de soies capillaires et les faisceaux dorsaux et ventraux sont constitués de soies du même type; il s'agit de crochets bifides (jamais pectinés) dont la dent distale est légèrement plus grosse et 


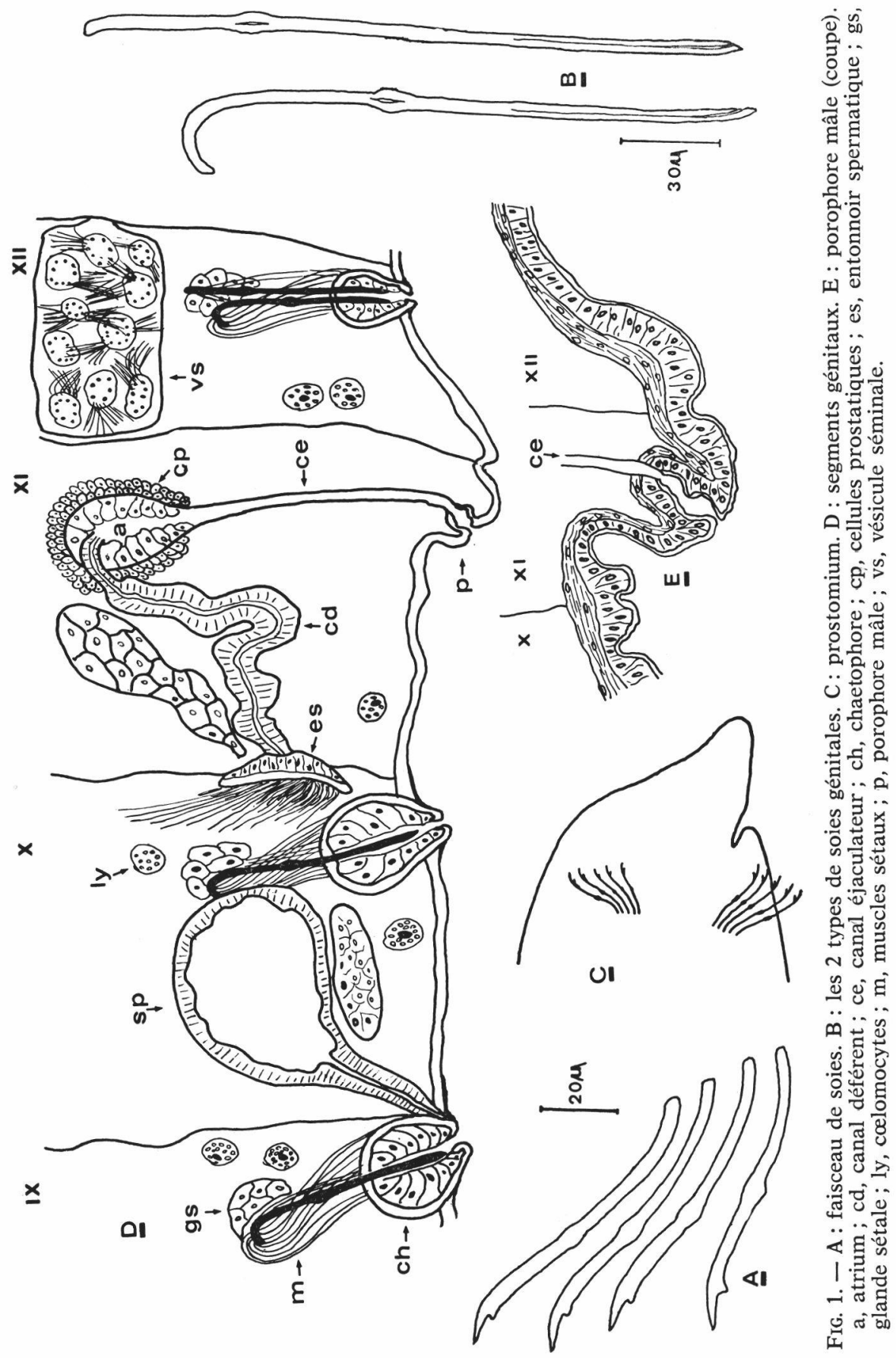


beaucoup plus longue que la dent proximale (fig. 1 A). La longueur des soies varie entre 80 et $100 \mu \mathrm{m}$; le nodulus, bien distinct, se situe aul $1 / 3$ ou aux $2 / 5$ de l'extrémité.

Les faisceaux dorsaux de la région préclitellienne comportent de 4 à 6 crochets; ceux de la région postclitellienne en ont généralement 2 (rarement 1) dans la zone moyenne et 3 (exceptionnellement 4) dans les tout derniers segments.

Les faisceaux ventraux comportent 5 ou 6 soies (rarement 4 ou 7) dans la région préclitellienne. Ils en ont 2 ou 3 seulement après le clitellum; les faisceaux à trois soies se rencontrent surtout dans les derniers segments.

- Soies copulatrices (fig. 1 B)

Il n'y a pas de soies péniennes en XI et les soies normales disparaissent à maturité. Par contre les soies ventrales des segments IX, X et XII sont modifiées sur les individus sexuellement mûrs : il y a une ou deux soies par faisceau, minces (moins de $5 \mu \mathrm{m}$ de diamètre) et longues de 220 à $240 \mu \mathrm{m}$, soit plus du double des soies normales. Elles sont excavées dans leur partie distale et possèdent donc un sillon longitudinal médian. Les bords restent à peu près parallèles bien que le sillon soit plus marqué vers l'extrémité.

Selon la forme de l'extrémité proximale et la position du nodulus on peut distinguer deux sortes de soies: les unes, présentes sur les 3 segments, ont une base très fortement recourbée et un nodulus net situć au $1 / 3$ ou aux $2 / 5$ de la partie proximale; les autres, parfois absentes sur certains segments, ont une hampe droite et un nodulus moins marqué et situé près de la base $(1 / 4$ à $1 / 6)$.

\section{LA RÉGION GÉNITALE}

Le clitellum n'est pas surélevé ; il débute au niveau des soies $\mathrm{X}$ et occupe la totalité des segments XI et XII.

La face ventrale possède 7 protubérances très caractéristiques. Il existe tout d'abord un porophore mâle unique, au niveau du seg. ment XI mais qui se prolonge sur le segment XII. Ce porophore $(0,1 \mathrm{~mm}$ de haut et $0,03 \mathrm{~mm}$ de long) est conique et nettement dirigé vers l'avant; il porte à son extrémité les deux pores mâles qui sont ainsi rapprochés de la ligne médiane.

De plus, les soies copulatrices sont implantées au milieu de chaetophores (au sens de Bouché, 1972) proéminents. En IX et X les 4 chaetophores sont très développés et placés dans l'alignement des faisceaux ventraux; en XII, ils sont plus réduits et plus écartés car ils sont repoussés vers l'extérieur par le porophore mâle.

Les pores des spermathèques sont situés entre les chaetophores (mais légèrement en arrière) des soies copulatrices du segment IX, près de l'axe du corps. Ils sont très légèrement proéminents. 


\subsection{Anatomie}

\section{CELomocytes}

La cavité cœlomique contient de très nombreux cœlomocytes sphériques et très granuleux; leur diamètre varie entre 15 et $30 \mu \mathrm{m}(22 \mu \mathrm{m}$ en moyenne).

\section{Appareil génital ( $f i g .1 \mathrm{D}$ )}

Il existe une paire de spermathèques localisées en $\mathrm{X}$. Chacune est formée par une ampoule légèrement ovoïde prolongée par un canal bien différencié qui débouche au contact du dissépiment IX/X. L'ampoule mesure 160 à $190 \mu \mathrm{m}$ de longueur, 145 à $160 \mu \mathrm{m}$ de largeur et sa paroi a une épaisseur de 20 à $30 \mu \mathrm{m}$; le canal, long de 140 à $160 \mu \mathrm{m}$, a un diamètre de 35 à $40 \mu \mathrm{m}$.

Nous n'avons jamais constaté la présence de spermatophores; le sperme est agglutiné en amas diffus à l'intérieur de la spermathèque.

Les conduits déférents mâles débutent chacun par un large pavillon cilié s'ouvrant sur le septum X/XI. Les spermiductes sont relativement courts : 270 à $300 \mu \mathrm{m}$ environ; ils ont une section homogène : 33 à $38 \mu \mathrm{m}$ de diamètre; l'épaisseur de la paroi est de $12 \mu \mathrm{m}$.

Deux atriums de forme ovoïde sont présents dans le segment XI ; leur longueur varie de 120 à $135 \mu \mathrm{m}$ et leur largeur de 60 à $75 \mu \mathrm{m}$. Ils sont recouverts par une couche de cellules prostatiques épaisse de 18 à $30 \mu \mathrm{m}$. Les spermiductes pénètrent subapicalement dans les atriums.

Chaque atrium se prolonge par un long canal éjaculateur très grêle : 180 à $260 \mu \mathrm{m}$ de longueur pour un diamètre de 9 à $12 \mu \mathrm{m}$. Ce canal, dépourvu de cellules prostatiques se recourbe vers l'avant au niveau du porophore mâle où il débouche dans une cavité formée par une invagination du tégument (fig. $1 \mathrm{E}$ ). Ce canal éjaculateur confère à l'atrium un aspect longuement pédonculé très caractéristique.

\section{STRUCTURE DES FOLLICULES SÉTIGÈres}

Chaque follicule est constitué par une aire glandulaire distale (chaetophore) piriforme qui détermine les proéminences visibles extérieurement. Cette aire glandulaire entoure l'orifice de sortie de la soie; celle-ci, engainée dans sa musculature pénètre longuement dans la cavité générale. A la base de chaque soie on trouve une glande sétale qui se colore moins bien que les chaetophores par l'hématoxyline.

En IX et X la hauteur totale du follicule est de 260 à $300 \mu \mathrm{m}$; dans le segment XII elle ne dépasse pas $220 \mu \mathrm{m}$. En IX et X, les chaetophores, très développés, ont une hauteur de 110 à $150 \mu \mathrm{m}$ pour un diamètre maximum de 95 à $120 \mu \mathrm{m}$; dans le segment XII ils sont plus réduits : 80 à $90 \mu \mathrm{m}$ de hauteur pour un diamètre de 70 à $80 \mu \mathrm{m}$. 


\section{3. - DISCUSSION}

- De par la nature des soies et la structure de l'appareil génital (une paire de spermathèques en $\mathrm{X}$, atrium en XI) cette espèce appartient à la famille des Tubificidae.

- L'abondance des cœlomocytes, l'absence de spermatophores et la prostate diffuse nous conduisent à ranger cette forme dans la sousfamille des Rhyacodrilinae telle qu'elle a été définie par Hrabe (1963) et revue par Brinkhurst et al. (1971).

- La présence de spermathèques et de cellules prostatiques, ainsi que la forme des atriums dans lesquels les spermiductes pénètrent subapicalement réfutent l'appartenance de cette espèce aux genres Paranadrilus, Epirodrilus, Bothrioneurum, Monopylephorus et Joly. drilus. Enfin, elle se distingue du genre Rhyacodrilus par:

- son atrium longuement pédonculé : canal éjaculateur grêle et plus long que l'ampoule ;

- l'absence de soies péniennes, généralement présentes chez les Rhyacodrilus ;

- la présence de soies spermathécales toujours absentes chez les représentants du genre Rhyacodrilus ;

- la présence d'un porophore mâle, jamais signalé sur les Rhyacodrilinae.

Sur l'ensemble de ces caractères nous avons décidé de créer le genre Protuberodrilus; ce nom fait référence aux 7 protubérances des chaetophores et du pore mâle. Cette espèce, Protuberodrilus tourenqui est amicalement dédiée à notre collègue J. N. TOURENo.

\section{Remarques}

- Il est intéressant de noter, chez $P$. tourenqui, la présence de chaetophores très développés; ces derniers sont très peu répandus chez les Tubificidae; ils sont cependant connus chez Monopylephorus lacteus (Smith, 1900), Limnodriloides barnardi Cook 1974, L. winckelmanni Michaelsen 1914 et Torodrilus lowryi Cook 1970.

- Chez les Tubificidae, ce sont généralement les soies péniennes (XI) et/ou spermathécales (X) qui peuvent être modifiées. Il convient de souligner, chez $P$. tourenqui, la présence de soies copulatrices sur le segment IX (déjà observées chez $M$. lacteus et $T$. lowryi) et surtout sur le segment XII. C'est la première fois que des soies copulatrices sont observées sur un segment post-atrial chez les Tubificidae. 


\section{TRAVAUX CITÉS}

BouchÉ (M. B.). 1972. - Lombriciens de France. Ecologie et Systématique. Annales de Zoologie-Ecologie animale, numéro hors série, $671 \mathrm{p}$.

Brinkhurst (R. O.) et JAMIESON (B. G. M.). 1971. - Aquatic Oligochaeta of the World. Oliver and Boyd. Edinburgh, $860 \mathrm{p}$.

Hrabe (S.). 1963. - On Rhyacodrilus lindbergi n. sp., a new cavernicolous species of the family Tubificidae (Oligochaeta) from Portugal. Bol. Soc. Portug. Cienc. Nat., $10: 52-56$.

Février 1980. 